\title{
CONTRIBUIÇÕES DA EXTENSÃO UNIVERSITÁRIA NA IMPLANTAÇÃO DO PLANO DE GERENCIAMENTO DE RESÍDUOS
}

Originais recebidos em: 22/09/2010

Aceito para publicação em: 13/01/2012

\section{Sonia Maria Isabel Lopes Ferreira} Universidade Estadual de Santa Cruz soniamilf@yahoo.com.br

Noélia Silva Oliveira
Universidade Estadual de Santa Cruz
noeliaoliveira04@gmail.com

\section{Érica Mascarenhas Andrade Santos \\ Santa Casa de Itabuma erika.andrade55@gmail.com}

\begin{abstract}
Resumo
O gerenciamento de resíduos sólidos de serviços de saúde, quando feito inadequadamente, oferece risco potencial ao ser humano e ao meio ambiente. As instituições que geram esses resíduos devem, por meio das características, do volume e tipo do lixo produzido, elaborar um Plano de Gerenciamento de Resíduos de Serviço de Saúde (PGRSS), que é um regulamento técnico elaborado pela ANVISA e aplicado a todos os geradores de resíduos de serviço de saúde, com a finalidade de estabelecer, em cada etapa do sistema, procedimentos detalhados de ações para um manejo seguro. $\mathrm{O}$ presente artigo trata de um relato de experiência que tem o objetivo de apresentar as contribuições dos Projetos de Extensão: Prevenção e Controle de Infecção Relacionada à Assistência à Saúde e Educação Continuada, da Universidade Estadual de Santa Cruz (UESC), em Ilhéus/BA, na elaboração e implantação do PGRSS de um hospital do município de Itabuna/BA, no período de 2008 a 2009. A metodologia de ação dessa atividade de extensão foi fundamentada nas normas federais vigentes, como a ANVISA RDC 306/2004 e o CONAMA 358/2005, além de rigorosa busca em base de dados, leitura e discussão entre os participantes sobre $\mathrm{o}$ assunto. Apesar das conquistas alcançadas com a elaboração do PGRSS, o hospital pesquisado encontra inúmeras dificuldades para avançar na implementação do plano, necessitando de melhoria nas instalações físicas, aquisição de materiais e equipamentos, amplo envolvimento técnico, administrativo e político. A participação de docentes e acadêmicos permitiu à universidade socializar e democratizar
\end{abstract}

conhecimentos, além de preparar profissionais capazes de atender às reais necessidades da população.

Palavras-chave: Gerenciamento de resíduos de serviços de saúde; Plano de Gerenciamento de Resíduos de Serviços de Saúde; Meio ambiente.

\section{CONTRIBUTIONS OF UNIVERSITY \\ EXTENSION IN THE DEPLOYMENT OF WASTE MANAGEMENT PLAN}

\section{Abstract}

The solid waste management services to health when done improperly offers potential risk to humans and the environment. The institutions that generate these wastes should, by the characteristics, volume and type of waste produced, and develop a Plan of Waste Management of Health Service (PGRSS), which is a tecnical regulation, prepared by ANVISA, applied to all waste generators in the health service, aiming to establish in each stage of the detailed procedures of actions for a safe handling. This article deals with an experience that aims to present the contributions of Extension Projects: Prevention and Infection Control Related to Health Care and Continuing Education of the Universidade Estadual de Santa Cruz - (UESC) Ilheus-Ba in PGRSS development and deployment of a county hospital Itabuna-Ba in the period 2008-2009. The methodology of action of this outreach activity was based on current federal standrads, such as the DRC ANVISA CONAMA $306 / 2004$ and $358 / 2005$, and rigorous search in the database, reading and discussion among participants on the subject. Despite the achievements in the development of PGRSS, the Hospital found numerous difficulties to advance the implementation of the plan, requiring improvements in physical facilities, purchase of materials and equipment, extensive technical involvement, administrative and political. The participation of teachers and students enabled the University to socialize and democratize knowledge, to prepare professionals capable of meeting the real needs of the population.

Keywords: Waste management of health services; waste management plan for health care; environment. 


\section{INTRODUÇÃO}

Os resíduos sólidos são hoje um grande desafio para a comunidade, principalmente para as instituições geradoras desses resíduos, que têm a competência de minimizar e gerenciar adequadamente o "lixo", a fim de evitar contaminação e impactos no meio ambiente. Entre as fontes de degradação ambiental, os resíduos gerados nos serviços de saúde apresentam uma peculiaridade importante: quando gerenciados inadequadamente, oferecem risco potencial ao ser humano e ao ambiente.

A Resolução $\mathrm{n}^{\mathrm{o}}$ 358, de 2005, do Conselho Nacional de Meio Ambiente (CONAMA), define esses resíduos como aqueles resultantes de atividades relacionadas com o atendimento à saúde humana ou animal, inclusive de assistência; laboratórios analíticos de produtos para a saúde; necrotérios; funerárias e serviços nos quais se realizem atividades de embalsamento; serviços de medicina legal; drogarias e farmácias, inclusive as de manipulação; estabelecimentos de ensino e pesquisa na área de saúde; centros de controle de zoonoses; distribuidores de produtos farmacêuticos; unidades móveis de atendimento à saúde; serviços de acupuntura, entre outros similares que, por suas características, necessitam de processos diferenciados em seu manejo, exigindo ou não tratamento prévio para sua disposição final.

O gerenciamento de resíduos sólidos, técnica utilizada para preservação da saúde pública e do meio ambiente, é definido pela Resolução RDC no 33/2003, da Agência Nacional de Vigilância Sanitária (ANVISA), como:

[...] um conjunto de procedimentos de gestão, planejados e implementados a partir de bases científicas e técnicas, normativas e legais, com o objetivo de minimizar a produção de resíduos e proporcionar aos resíduos gerados, um encaminhamento seguro, de forma eficiente, visando à proteção dos trabalhadores, à preservação da saúde pública, dos recursos naturais e do meio ambiente.

Através de um regulamento técnico elaborado pela ANVISA e aplicado a todos os geradores de resíduos de serviço de saúde (RSS), as instituições que geram esses resíduos deverão, por meio de características, tais como volume e tipo do lixo produzido, elaborar um Plano de Gerenciamento de Resíduos de Serviço de Saúde (PGRSS), que tem como finalidade estabelecer procedimentos detalhados de ações para um manejo seguro em cada etapa do sistema: geração, classificação, segregação, acondicionamento, transporte, armazenamento, tratamento e disposição final, bem como 
treinamento para o manejo dos resíduos e utilização adequada de equipamentos de proteção individual (EPI).

O PGRSS tornou-se exigência legal, como documento integrante do processo de licenciamento ambiental, através da Resolução no 5 , de agosto de 1993, do Conselho Nacional de Meio Ambiente (CONAMA), que estabelece a obrigatoriedade de sua elaboração, assim como a sua aprovação junto ao órgão ambiental competente. Essa Resolução, assim como a Resolução CONAMA no 283/01, que a complementa, regulamenta o "tratamento e a disposição final adequada" dos resíduos de serviços de saúde, visando "prevenir danos à saúde e ao meio ambiente".

As vantagens do desenvolvimento e aplicação do Plano de Gerenciamento de Resíduos são: redução de riscos ambientais, redução do número de acidentes de trabalho, redução dos custos de manejo dos resíduos, incremento da reciclagem e redução do número de infecções hospitalares relacionadas ao manejo incorreto dos resíduos.

O presente artigo trata de um relato de experiência que tem o objetivo de apresentar as contribuições dos Projetos de Extensão: Prevenção e Controle de Infecção Relacionada à Assistência à Saúde e Educação Continuada, da Universidade Estadual de Santa Cruz (UESC), em Ilhéus/Bahia, na elaboração e implantação do PGRSS de um hospital do interior da Bahia no período de 2008 a 2009.

A necessidade de observância rigorosa das técnicas corretas de manejo dos resíduos de serviços de saúde mostra a relevância dessa ação de extensão pela importância de garantir a segurança de funcionários, pacientes e visitantes desses serviços, além de protegerem a comunidade e o meio ambiente.

Desde 2003 os Projetos Prevenção e Controle de Infecção Relacionada à Assistência à Saúde e Educação Continuada atuam junto a Comissão de Controle de Infecção Hospitalar (CCIH) e Serviço de Educação Continuada desse hospital, cuja integração tem contado com a participação efetiva de professores e acadêmicos, possibilitando a interface com o ensino através da flexibilização curricular, com a pesquisa e a divulgação e publicação de produções científicas. 


\section{METODOLOGIA}

O hospital onde foram desenvolvidas as atividades está situado no município de Itabuna, na região Sul da Bahia, localizado a 432,9 Km de Salvador, com uma população aproximada de 200 mil habitantes (IBGE, 2010). Trata-se de uma instituição filantrópica, de grande porte, que possui 198 leitos e presta atendimento ao SUS e a convênios particulares. É centro de referência para atendimento em alta complexidade em oncologia, neurologia, clínica médica e cirúrgica a pacientes adultos e idosos. Serve como campo de prática para os cursos de medicina e enfermagem da UESC.

A metodologia de ação dessa atividade de extensão foi fundamentada nas normas federais vigentes, como a ANVISA RDC 306/2004 e o CONAMA 358/2005, além de rigorosa busca em base de dados, leitura e discussão entre os participantes sobre o assunto. Após a fundamentação teórica, foi iniciada a elaboração do plano de gerenciamento de resíduos, que contou com a participação dos docentes coordenadores dos projetos, dois alunos bolsistas, e representantes da instituição nomeados pela provedoria: enfermeira e médico da $\mathrm{CCIH}$, técnico em segurança do trabalho, médico e enfermeira do programa de controle de saúde ocupacional.

Como não existe um modelo de PGRSS aplicável a todos os tipos de estabelecimentos, este pode apresentar uma variação de acordo com o tipo, estrutura e especialidade de estabelecimento. Assim, baseado nas exigências legais, principalmente nas Resoluções do CONAMA n $5 / 93$ e 283/01 e da ANVISA RDC 33/03, existem alguns passos que todo Plano deve contemplar. Dessa forma, com base nas legislações vigentes, foi possível determinar uma metodologia de elaboração do PGRSS de forma objetiva, possibilitando o entendimento e confecção de suas etapas. Após definição dos objetivos do Plano e da equipe de trabalho, seguiram-se as etapas descritas a seguir.

$1^{\circ}$ Passo - diagnóstico da situação. Foi elaborado um formulário, para coleta de dados, contemplando um inventário sobre os tipos e quantidade de resíduos gerados, e os tipos de riscos associados a cada um dos resíduos. Para conhecer a quantidade de resíduos gerados pelo hospital, elaborou-se uma planilha com os setores de geração de resíduos e os tipos de acondicionadores adotados para segregar seus resíduos. Essa planilha foi preenchida durante três semanas pelo funcionário responsável pela coleta dos resíduos. Os responsáveis pela elaboração do PGRSS acompanharam um dia inteiro de coleta, nos turnos da manhã e tarde, e identificaram a rota de coleta interna dos resíduos, a utilização ou não dos EPI pelo funcionário responsável pela coleta, os locais 
de armazenamento e os tipos de veículos utilizados na coleta interna dos resíduos. Foram levantados também dados sobre a limpeza, formação e capacitação dos funcionários envolvidos com o manejo dos resíduos; caracterização do estabelecimento (razão social, nome de fantasia, endereço completo, especialidades, capacidades, responsáveis); dados sobre o espaço físico (área do terreno, área construída); e os locais onde poderiam ocorrer os aspectos ambientais - processos ou atividades que causam impactos ao meio ambiente (resíduos sólidos, efluentes líquidos e gasosos). Todas as informações encontradas foram anotadas em formulários próprios e serviram para avaliar a situação em que o estabelecimento se encontrava antes da elaboração e implementação do PGRSS.

$2^{0}$ Passo - elaboração do PGRSS do hospital. Obedecendo-se aos critérios estabelecidos nas normas, foram elaboradas medidas para garantir a implementação e o cumprimento dos procedimentos definidos para o plano de gerenciamento de resíduos, ou seja, neste passo são registrados os procedimentos de manejo para cada categoria de resíduos, desde a segregação até a disposição final dos resíduos, o levantamento dos riscos associados ao manejo dos RSS. Dessa forma, a elaboração do PGRSS seguiu a normativa existente, compreendendo as etapas a seguir:

Classificação: a classificação dos resíduos nos permite tomar decisões quanto aos resíduos que deverão ser recuperados (reciclados) e quais poderão seguir seu fluxo para o tratamento e/ou disposição final. O estabelecimento deve definir, de acordo as resoluções vigentes, qual classificação deverá ser adotada.

Segregação: consiste em separar e colocar os resíduos no local designado no momento e local de sua geração, com o objetivo de racionalizar e impedir a contaminação de grandes quantidades de lixo. Na descrição do procedimento de segregação e acondicionamento, o estabelecimento deve registrar como segrega e acondiciona seus resíduos.

Acondicionamento: deve estar de acordo com o tipo de resíduo, observando-se principalmente materiais cortantes e perfurantes. A exceção dos perfurocortantes, que devem ser descartados em recipientes rígidos, todos os resíduos sólidos devem ser acondicionados em sacos constituídos de material resistente à ruptura e vazamento, impermeável, baseado na NBR 9191/2000 da ABNT (Associação Brasileira de Normas Técnica), 
respeitados os limites de peso de cada saco, sendo proibido o seu esvaziamento ou reaproveitamento. Coleta: divide-se em interna e externa. A coleta interna consiste no recolhimento do resíduo da lixeira no local de produção, no fechamento do saco, e no seu transporte até a sala de depósito interno temporário do lixo (sala de resíduos ou material sujo). Os sacos/recipientes devem estar ocupados até $2 / 3$ de sua capacidade e, ao serem coletados, devem ser bem fechados. Não é permitido despejar o conteúdo de um saco no outro. Deve-se usar sempre equipamento de proteção individual. A coleta externa consiste no recolhimento do lixo temporariamente armazenado na unidade; os horários de coleta devem obedecer a uma programação que seja de conhecimento de todos, observando que o resíduo deve permanecer o menor tempo possível na unidade. Nesse procedimento, os estabelecimentos devem registrar os roteiros de coleta, os responsáveis pela coleta e transporte e os tipos de carros utilizados para o transporte.

Armazenamento: também é classificado como interno e externo. O armazenamento interno visa conter os resíduos gerados até sua coleta nos fluxos determinados em condições ambientais e ocupacionais satisfatórias. $\mathrm{O}$ armazenamento externo visa conter os resíduos enquanto aguardam a coleta municipal ou encaminhá-los para tratamento na própria unidade. Nessa fase, deve ser registrado como é realizado o armazenamento, além da descrição do espaço físico, que tem que estar de acordo com as normas da ABNT 12809, 12810/93.

Transporte: pode ser denominado interno, quando vai da unidade geradora até a sala de resíduos, ou externo, quando vai da sala de resíduo até o abrigo ou local de apresentação à coleta pública. Deve-se obedecer a um roteiro pré-estabelecido, de forma a impedir o cruzamento de material limpo com os resíduos. O lixo deve ser transportado em veículos ou carrinhos dimensionados de acordo com o volume coletado.

Tratamento e disposição final: a responsabilidade sobre os resíduos fica dividida entre o estabelecimento gerador e o órgão público municipal. O tratamento é o processo físico, químico ou biológico dos resíduos, buscando torná-los menos agressivos à saúde pública e ao meio ambiente. A 
disposição final de cada fração, de acordo com suas características específicas, facilita o gerenciamento e reduz custos. Além de diminuir o seu período de risco, reduz a probabilidade de ocasionar doenças aos trabalhadores em contato direto ou indireto. No PGRSS devem ser descritos os tipos de tratamento utilizados pelo estabelecimento para cada tipo de resíduo e se ele é realizado pelo estabelecimento ou por empresa especializada. Outra informação que deve ser registrada é quanto ao Programa de Reciclagem utilizado pelo estabelecimento, no qual são definidas as ações quanto à utilização dos 3 Rs (reduzir, reutilizar e reciclar).

$3^{\circ}$ Passo - após elaboração, o PGRSS foi apresentado para a provedoria, direção administrativa, direção médica e coordenação de enfermagem. Após aprovação interna, foi encaminhado para avaliação das autoridades sanitária do município.

$4^{0}$ Passo - depois de confirmada a aprovação do PGRSS pelas autoridades sanitárias do município, o PGRSS foi divulgado para toda a comunidade hospitalar. Seguiu-se, então, para a fase de formação de grupos multiplicadores para a adequação das rotinas por parte dos funcionários e confecção de folhetos informativos. A educação continuada objetivava que a comunidade hospitalar conhecesse o sistema adotado para o Gerenciamento de Resíduos Sólidos de Serviços de Saúde, como os símbolos gráficos, padrões de cores adotados, horário e percurso de coleta dos resíduos, e localização do abrigo dos resíduos. Os profissionais envolvidos com a higiene e limpeza deveriam estar conscientes quanto à necessidade da utilização correta de equipamentos de proteção individual e manejo adequado dos resíduos. Além disso, deveria contemplar: noções gerais sobre ciclo de vida dos materiais, conhecimento da legislação em vigor, definições, tipo e classificação dos resíduos, formas de reduzir a geração, higiene pessoal e dos ambientes, providências em caso de acidentes e situações emergenciais (CAMARGO et al., 2009). Para desenvolver e elucidar a capacitação, o processo educativo foi fundamentado nas normas estabelecidas no PGRSS elaborado pelo grupo e nas orientações do Ministério da Saúde relativas à exposição ocupacional. A metodologia utilizada nessa capacitação foram aulas expositivas, problematização, entrega de folhetos informativos, treinamentos in loco nas unidades. Foi necessária, 
ainda, a aquisição dos equipamentos necessários para melhorar o gerenciamento dos RSS.

\section{RESULTADOS E ANÁLISE}

A enfermeira da $\mathrm{CCIH}$ foi designada pela diretoria para ser a responsável técnica pelo Plano de Gerenciamento de Resíduos do Hospital, com registro junto ao seu conselho de Classe.

Os resultados da caracterização média do hospital apontaram para uma geração diária de $270 \mathrm{~kg} /$ dia de resíduos ou, para os 198 leitos, $1,36 \mathrm{Kg} /$ leito/dia. Esse cálculo seguiu a descrição de Monreal (1993 apud SCHNEIDER, 2004) que descreve que, ao se calcular a geração de resíduos de um estabelecimento de saúde, deve-se adotar uma relação entre a quantidade média gerada por dia e o número de leitos ocupados. Em comparação com a geração média brasileira de resíduos de $2,63 \mathrm{Kg} /$ leito/dia, citado pelo mesmo autor, percebe-se que o hospital em estudo gera menos resíduos que a média nacional.

Adotou-se a classificação da RDC 306/04 que divide as classes de resíduos em Grupo A (potencialmente infectantes); Grupo B (químicos); Grupo C (rejeitos radioativos); Grupo D (resíduos comuns) e Grupo E (perfurocortantes).

A segregação dos resíduos e os acondicionadores seguiram a seguinte diferenciação:

- sacos brancos leitosos - utilizados para os resíduos com risco biológico (papel higiênico, curativos, equipos de soro, luvas, bolsas de sangue já utilizadas, sondas vesicais, nasogástricas, naseoentéricas, restos cirúrgicos, fraldas descartáveis usadas, etc.);

- caixas Descartex - utilizadas para os resíduos perfurocortantes (agulhas, ampolas, pipetas, lâmina de bisturi, lâmina de barbear, vidros quebrados ou que quebrem facilmente e seringas com agulhas);

- sacos pretos - utilizados para os resíduos orgânicos (papel carbono, papel toalha, restos de alimentos); sacos azuis, utilizados para os resíduos recicláveis (papel/papelão, vidros vazios, não quebrados e não perigosos, plásticos e metais, frascos de soro vazios e embalagens em geral). 
Com base nas resoluções, foram fixados procedimentos exigíveis para garantir condições de higiene e segurança no processamento interno de resíduos perigosos, especiais e comuns nos serviços de saúde. Todo recipiente tem que ser fechado de forma a não possibilitar vazamento e o fechamento deve ser feito quando $2 / 3$ de sua capacidade estiverem preenchidos; após o fechamento, o recipiente deve ser retirado imediatamente da unidade geradora.

$\mathrm{Na}$ etapa de armazenamento interno de resíduos, foi encontrada maior dificuldade, pois apenas uma unidade de internação dispõe de local apropriado para esse fim, ficando os recipientes acumulados no final de cada corredor, em área de grande trânsito de pacientes, profissionais e visitantes, comprometendo a higiene e a segurança do ambiente hospitalar. Segundo Obladen (1992), o local reservado para o armazenamento interno deve ser localizado o mais distante possível da área de circulação de pessoas, dispensas e cozinhas e possuir sistemas de trancas e placas de alerta especificando a natureza dos resíduos por motivos óbvios. O mesmo autor cita que esse local deve observar os seguintes padrões: piso e paredes de material liso, resistente e lavável, ralo ligado à rede de esgoto, ventilação adequada, telada e ponto de água. Esse local deve ser submetido à limpeza e desinfecção periódica e não menos que diariamente. Esse problema não foi encontrado apenas em setores como Unidade de Tratamento Intensivo (UTI), Hemodiálise e Centro Cirúrgico.

O hospital utiliza, na maioria das vezes, para acondicionamento de resíduos, recipientes inadequados: grande número deles não possui tampa. Os recipientes para acondicionamento dos resíduos devem ser: de tamanho, forma e material apropriado, de maneira a assegurar suficiente capacidade, resistência e integridade (RIBEIRO FILHO, 2000); fáceis de manusear e proporcionar, quando for o caso, limpeza e hermeticidade de acordo com os requisitos sanitários básicos; e acionados por pedal para evitar a exposição dos resíduos na unidade e prevenir acidentes e contaminação das mãos dos profissionais, ao descartarem os resíduos.

A coleta interna continuou sendo realizada pelos profissionais do Setor de Higienização. Anteriormente era realizada três vezes ao dia, mas foi identificada a necessidade de haver mais uma coleta no final da tarde, devido ao grande volume de resíduos gerado. Foi determinada uma nova rota para coleta de resíduos, visando a uma redução da circulação do carrinho de resíduos dentro dos corredores do hospital. 
A coleta externa dos resíduos é realizada por uma empresa terceirizada que possui licença ambiental para exercer tal atividade. Essa empresa coleta todos os resíduos gerados pelo hospital de segunda a sexta-feira, responsabilizando-se pelo tratamento e disposição final dos resíduos. No abrigo externo, os resíduos coletados dentro de seus acondicionadores são colocados em bombonas de 50 e 100 litros. Por dia são preenchidas 5 bombonas de 100 litros e 3 de 50 litros. O hospital paga R\$ 49,50 por cada bombona, tendo um gasto mensal de $\mathrm{R} \$ 11.880,00$ pela disposição final dos resíduos. Após a coleta, essa empresa compacta e incinera os resíduos, em Itabuna/BA, e transporta suas cinzas até a cidade de Camaçari/BA, onde são aterradas em sistemas de disposição final licenciados. Falqueto, Kligerman e Assumpção (2010) citam que é de responsabilidade das autoridades sanitárias do Ministério da Saúde, dos estados e municípios inspecionar periodicamente essas empresas, garantindo a proteção da saúde e do meio ambiente.

O abrigo externo de resíduos possui: revestimento cerâmico nos pisos e paredes, facilmente laváveis; ralos sifonados, para drenagem das águas provenientes da lavagem; e ponto de água e luz próximo e coberto por telhas de fibrocimento.

Todos os funcionários do hospital, familiares e os acompanhantes dos pacientes foram treinados para segregar adequadamente os resíduos e reconhecer os sistemas de identificação. Essa capacitação tem ocorrido de forma continuada, buscando não apenas a capacitação em procedimentos técnicos específicos sobre o manejo dos RSSS, mas, fundamentalmente, despertar a consciência coletiva quanto à responsabilidade com a própria vida humana e com o meio ambiente, obtendo novos valores e, com isso, reduzir a geração de resíduos e gerenciar efetivamente seu manuseio.

A esse respeito, Doi e Moura (2011), após investigar os conhecimentos e atitudes dos profissionais frente ao descarte dos RSS, destacam a importância de um processo educativo que leve à reflexão das práticas atuais por parte dos profissionais e, concomitantemente, comprometimento com a busca e adesão às soluções de atitudes desejáveis com o descarte dos RSS. Por sua vez, Camponogara, Ramos e Kirchhof (2009) evidenciam, com base nos achados do estudo sobre reflexividade ecológica no contexto do trabalho hospitalar e sua influência sobre a ação laboral dos trabalhadores, que o trabalhador hospitalar, embora reflexivamente afetado pela atual problemática ecológica, age em prol da preservação ambiental predominantemente pautado em preceitos normativos. Nesse caminhar, face à inexistência de uma discussão 
aprofundada sobre o assunto, o desenvolvimento de ações fundamentadas em um sentimento de responsabilidade ambiental, no contexto do trabalhador, fica prejudicado, tornando-se importante haver espaço para um processo educativo sobre o tema.

O PGRSS elaborado pela equipe designada pelo hospital, em conjunto com os docentes e discentes dos projetos de extensão, foi aprovado pelos órgãos sanitários do estado e município e, após esses trâmites legais, foi adotado e implementado na instituição. Apesar disso, o hospital necessita cumprir algumas exigências, tais como: adquirir lixeiras com pedal para lixos do grupo A e reposição das que se encontram danificadas; adquirir caixas Descartex para resíduos do grupo E, pois o Hospital tem improvisado o uso de recipiente de parede rígida, porém inadequado, que pode colocar em risco a segurança dos profissionais; adquirir containers para acondicionamento dos resíduos; e construir abrigo interno em alguns setores.

É importante destacar que as medidas propostas precisam ser constantemente reavaliadas, mantendo-as sempre atualizadas pelas normas vigentes e preservando a conscientização e participação de toda a comunidade hospitalar. Dessa forma, o gerenciamento correto dos resíduos sólidos tem proporcionado para o hospital em estudo e para a saúde pública: (i) a otimização de vários aspectos benéficos sanitários e ambientais; (ii) reaproveitamento e reciclagem de materiais; (iii) minimização de espaço para aterros sanitários, com redução de custo; (iv) redução de infecção hospitalar; (v) redução de acidentes ocupacionais; (vi) menos perigo de contaminação do meio ambiente.

Faz-se necessário, também, o envolvimento integrado da Gestão Municipal para dar solução a vários pontos polêmicos dentro da norma que são de competência dos órgãos estaduais e/ou municipais para discutir práticas corretas e viáveis para o município.

Houve evoluções bastante significativas nesse ambiente, como: melhor organização dos setores, conscientização da equipe de enfermagem e higienização, e redução do risco de acidentes de trabalho, além de facilitar a coleta e ajudar o meio ambiente. Contudo, para a continuidade dessas conquistas, é importante que a instituição hospitalar tenha a preservação ambiental como política institucional, e que esse tema seja colocado na pauta de discussão do processo de educação permanente dos trabalhadores. 


\section{CONSIDERAÇÕES FINAIS}

Consideramos que, somente por meio da implementação e cumprimento do PGRSS, e do acompanhamento de suas etapas operacionais, de acordo com as leis vigentes, podemos evitar a contaminação do meio ambiente e diminuir custos para a instituição. Observou-se, portanto, que a implementação do PGRSS necessita de amplo envolvimento técnico, mas tornar-se-á inviável sem um adequado empenho administrativo e político. O correto manejo dos RSS contribui para minimizar riscos ocupacionais, ambientais e de infecção hospitalar. A educação continuada contribui para o despertar dos profissionais, que precisam estar conscientes da importância de seu papel em todo o processo do PGRSS. A participação de docentes e acadêmicos permite à universidade socializar e democratizar os conhecimentos dos diversos cursos e também preparar seus profissionais, não somente com a estratégia do ensinotransmissão, mas complementando a formação com a estratégia do ensino-aplicação. Os cenários da prática de integração ensino-serviço nos serviços de saúde e na comunidade promovem a inter-relação educação (UESC) e trabalho (serviços de saúde), procurando contribuir com a efetiva (re)organização e implementação das diretrizes do SUS e da necessidade de preparar profissionais capazes de atender às reais necessidades da população.

\section{REFERÊNCIAS}

ASSOCIAÇÃO BRASILEIRA DE NORMAS TÉCNICAS. ABNT. NBR 12808: Resíduos de Serviços de Saúde: Classificação. 1993.

Janeiro, 1993.

NBR 12809 - Manuseio dos resíduos de serviços de saúde. Rio de . NBR 12810 - Coleta de resíduos de serviços de saúde, 1993.

. NBR 9191- Acondicionamento de resíduos de serviços de saúde, 2000.

BRASIL. Agência Nacional de Vigilância Sanitária. Resolução RDC no 33, 25 de fevereiro de 2003. Brasília: ANVISA, 2003. 
Agência Nacional de Vigilância Sanitária. Resolução RDC no 306, 7 de dezembro de 2004. Brasília: ANVISA, 2004.

BRASIL. Conselho Nacional do Meio Ambiente. Resolução $\mathbf{n}^{\mathbf{0}}$ 05, de 5 de agosto de 1993. Diário oficial da República Federativa do Brasil, Brasília, 5p.

.Conselho Nacional do Meio Ambiente. Resolução no 283, de 12 de julho de 2001. Diário oficial da República Federativa do Brasil, Brasília, 4p.

. Conselho Nacional do Meio Ambiente. Resolução n⿳358, de 29 de abril de 2005. Diário oficial da República Federativa do Brasil, Brasília, 5p.

CAMARGO, M.E.; MOTTA, M.E.V.; LUNELli, M.O.; SEVERO, E.A. Resíduos Sólidos de Serviços de Saúde: um estudo sobre o gerenciamento. Rev. Scientia Plena. Vol. 5, NUM.7. 2009.

CAMPONOGARA, M.S.; RAMOS, F.R.S.; KIRCHHOF. Um olhar sobre a interface trabalho hospitalar e problemas ambientais. Rev. Gaucha Enferm., Porto Alegre (RS) 2009 dez. 30(4): 724-31.

DOI, K.M.; MOURA, G.M.S.S. RESÍDUOS SÓLIDOS DE SERVIÇOS DE SAÚDE: uma fotografia do comprometimento da equipe de enfermagem. Rev. Gaucha Enferm., Porto Alegre (RS) 2011 jun;32(2):338-44.

FALQUETO, E.; KLIGERMAN, D.C.; ASSUMPÇÃO, R. F. Como realizar o correto descarte de medicamento? Rev. Ciência \& Saúde Coletiva, 15(Supl.2): 3283-3293 2010 .

INSTITUTO BRASILEIRO DE GEOGRAFIA E ESTÁTISCA (IBGE). Pesquisa de Saneamento Básico do Município de Itabuna-Ba. 2010.

(a) OBLADEN, N.L. Conceitos gerais e aspectos sanitários dos resíduos hospitalares (resíduos de serviços de saúde- RSS). In: Seminário Internacional sobre manejo disposicion de Resíduos Hospitalares, p.2-23. 1992.

RIBEIRO FILHO, V.O. Gerenciamento de Resíduos de Serviços de Saúde. In: Infecção Hospitalar e suas Interfaces na Área da Saúde. São Paulo: Editora Atheneu. 2000.

SCHNEIDER, V.E. ET AL.. Manual de Gerenciamento de resíduos sólidos de serviços de saúde. 2 ed. Ed., Caxias do Sul, Educs. 2004 